\title{
Abstract
}

\section{Dead body management following disasters: Capacity assessment of District General Hospital, Trincomalee}

\author{
Nayani Rajapaksha ${ }^{1}$, Dinesh Fernando ${ }^{2}$, Muralli Vallipuranathan ${ }^{3}$ \\ ${ }^{1}$ Postgraduate Institute of Medicine, University of Colombo, ${ }^{2}$ Faculty of Medicine, University of Peradeniya, ${ }^{3}$ Ministry \\ of Health, Sri Lanka
} Key words: disaster, capacity, deceased management, institutional capacity assessment,
capacity development

\section{Introduction}

This study intended to assess the capacity within the District General Hospital, Trincomalee in the management of dead-bodies following disasters.

\section{Methods}

A cross-sectional survey was carried out among health care workers to assess their individual capacity using self-administered pre-tested tool. A proportionately stratified sample of 165 health workers were taken as the study population which consisted of 145 doctors, 195 nursing officers and 197 minor employees. The organizational capacity was assessed by the key informants from disaster management committee using a self-guided pre-tested tool in 2014. Data was analysed using SPSS version 21.

\section{Results}

Among the study sample, 118 (75.2\%) had more than average knowledge on deceased management. There was a statistically significant mean knowledge difference between the selected three groups of workers. There were positive attitudes among them. Only 3.8\% had undergone training programs related to dead body maangement. Lack of training can be associated with the reduction of the capacity to handle disaster situations.

\section{Discussion}

There was a clear need to increase the capacity in the areas of financial allocation and staff of dead body management team. There was a high level of capacity in the areas of planning for deceased management and the community participation in the event of a disaster. Results concluded that, the overall capacity for dead-body management following disasters at DGH Trincomalee needs to be improved by conducting capacity development programmes. 